\title{
QUANTITATIVE ASPECTS OF
}

\section{THE MANAGEMENT OF HEALTH INFORMATION}

\author{
by
}

\section{Leo Egghe}

LUC, Universitaire Campus, B-3590 Diepenbeek, Belgium ${ }^{(*)}$

and

UIA, Universiteitsplein 1, B-2610 Wilrijk, Belgium.

\begin{abstract}
We report on quantitative techniques for managing health information as well as for the marketing and the management of health libraries and other health information centres.

Important for marketing and management of libraries are the p.r. (public relations) and p.a. (public awareness) activities. We discuss reports (e.g. case studies, annual reports, ...) as important tools in this and pay special attention to the case of health information centres.

We go into quantitative (mainly statistical) techniques in order to gather and manage data that form the basis of such reports : data context and definition, data collection, examples and its use as complete or incomplete sets : descriptive methods (such as graphs, regression, measures of central tendency and dispersion, etc...), sampling, confidence intervals and hypothesis testing. We stress the fact that any librarian can become conversant with such analysis techniques and we underline the importance of such activities, both in libraries and in the population of its users.
\end{abstract}

We give an account on some biomedical exercises that were carried out in LUC and in a Flemish interlibrary context.

Finally, also the health literature itself can be the topic of quantitative investigations. We describe aspects of citation analysis with applications in the areas of the evaluation of medical research and aspects of bibliometric analysis, such as (e.g.) ageing, growth and collaboration.

Key words and phrases : management, report, statistics, citation analysis, bibliometrics

$\left(^{*}\right)$ Permanent address. 


\section{〈heading 1)I. Introduction}

It is a quite obvious remark that, nowadays, services (such as libraries) must continuously prove themselves : prove that one is still needed and even that one performs better with shrinking budgets. This is the more true with libraries, being services that do not exist "for their own sake" (i.e. they are not money making companies on their own : on the contrary : they cost (lots of) money). As far as I can see this, it is even the more true for medical libraries where its users - in general - are not very "library minded". Some people even think that the role of (mainly scientific) libraries is almost over. This is not true ; the opposite is more true !

We must be able to convince subsidising leading bodies that libraries play an increasingly important role in modern scientific life. This "proof" can be given by establishing p.r. and p.a. activities (p.r. = public relations, p.a. = public awareness). P.r. means : making libraries and its services attractive ("sell" your services) and publicising the library ; p.a. means : informing directors, making them aware of activities and needs of the library and make sure that libraries are in their minds when they have to make (budgettary) decisions.

The physical form of p.r. and p.a. actions are diverse reports of several types :

p.r. : informative brochures, guides, folders, WWW pages in an attractive form, press releases (via the organisation of press conferences),

p.a. : special reports prepared for discussions in meetings, annual reports.

Of course, in some cases annual reports do also serve as p.r. tools, especially in the case of company information.

All these p.r. and p.a. actions do not only serve the goal of informing the subsidising leading bodies : they are also needed to inform the library users. Giving them good information leads to an understanding of certain rules or to the acceptance of the fact that one is obliged to ask a fee for certain services.

Last but not least, reports are also needed for the library management itself : only by producing professional reports the librarian and his/her staff are able to fully "understand" what is going on in the library and to predict future uses and problems.

Reports can only be produced in a professional way by collecting concrete and sufficient "hard" correct data. Collecting such data is very well possible in the everyday life of a library but its complexity is sometimes underestimated. Important in this matter is knowing the context (universe) about which one wants to report about and secondly is the exact definition of the property under study. We go into these matters in the next section. Also many examples will be given.

In the third section we discuss aspects of the use of these "raw" data in order to make them useful in reports. Here we discuss complete data as well as incomplete data (i.e. samples) and explain how conclusions can be drawn from these two different types of data. Some biomedical "exercises" are presented. 
The last section indicates briefly some applications (in biomedical areas) of bibliometric (scientometric) aspects such as citation analysis, growth, obsolescence (or ageing) and collaboration.

It is clear that all the techniques mentioned above are applicable in the general area of information science (hence in particular in any library). Therefore we present them in a general context. But whenever it is possible we will give applications in the area of health information sciences. It will become clear that health libraries occupy a special place amongst the scientific libraries and hence it is useful to highlight this special situation in a professional way.

〈heading 1)II. Data

〈heading 2)II.1 Context of data

One must know from which "universe" one wants to extract data, i.e. one must decide on what universe (the total population) one wants to report on. Let us give some examples :

\section{PROPERTY}

number of circulations

prices of books

OPAC use

delivery times of books at a bookseller or of interlibrary material by a library

users' satisfaction

number of authors of medecine books

\section{UNIVERSE}

library (I)

library (I or NI)

at a bookseller (NI)

worldwide (NI)

library (I)

network (I)

bookseller (NI)

used library (NI)

users of the library (NI)

library (I or NI)

worldwide (NI)

library (NI)

Here "I" means "integral counts" and "NI" means "non integral counts". The former means that we are able to check every single element in the universe (also called population) and we can give exact conclusions on the property in this universe. The latter means that we do not have time or money enough to check every single element in the investigated universe (or that it is even impossible to do so) and hence that we have to content ourselves with a sample. In this case we cannot expect to draw $100 \%$ sure conclusions for the investigated property in this universe. We go into these matters in the sequel. 
It is obvious that the investigated properties must be clearly defined. This clarity must be in our own mind but also in the minds of the other library staff. Otherwise different staff members in the same library report differently on the same topics, hence making the outcome useless. Let us give two examples of possible confusions :

- $\quad$ "Money spent for books" is ambiguous unless specified : are we interested in the budgetted amount, the amount of money appearing in the orderings, the total price of the delivered books, the toal amount of the invoiced books or the total amount paid for books in a certain year? Is the definition of "book" clear (e.g. does it include serials, ...)?

"Number of circulations" : does it also contain continuations, interlibrary circulations and so on?

A clear definition of the investigated properties is the more necessary in case (and this is strongly advisable) we want to make common (or comparable) reports amongst similar libraries (e.g. scientific libraries or only university libraries, public libraries, governmental libraries, business libraries, and so on). Many meetings between the colleagues are necessary in order to have a common view and understanding of the topics one wants to report on.

Common or comparable reports amongst similar libraries are very useful in case a single librarian wants to prove that his/her library is not receiving enough budgets/manpower as compared to the other ones in the same consortium.

\section{〈heading 2)II.3 Organisation of the data gathering}

Data are collected on a yearly basis (usually) as they have to be used e.g. in the annual report. Most commonly this year on which one reports is a calendar year. Even libraries of academic institutions (universities or other schools where professional education is given) do report on this basis due to the fact that subsidies are allocated on this basis.

Don't be mistaken : collecting data needs a continuous effort throughout the year (e.g. how many books have been reshelved, how many visitors were there, time spent in cleaning the library and so on). Only in the case data are generated by a computer, they can be produced in the beginning of the next year (e.g. number of circulations, number of books catalogued, and so on). In all the other cases one needs to be prepared by organising a kind of "logbook" (e.g. for counting the number of books reshelved or for counting the number of library visitors) or a file (e.g. of invoices, ...). From the above it is clear that, along with the dicussions on automating the library, one should always keep in mind the issue of the automatic generation of library statistics.

What needs to be collected? This is not an easy question. It depends on the type of library and even of the local situation. One needs to collect data on these topics that are important for allowing the librarian to prove certain things (i.e. to support certain claims). In any case : better collect few data if they can be collected in an accurate way than to collect more inaccurate data. We repeat here that every library staff member must have the same insight in the very nature (definition) of the wanted data. The same goes for the different libraries 
in a consortium. A good example are the ARL statistics (ARL = American Research Libaries, mostly university libraries in North-America), collecting only 33 "basic" statistics (see [1]).

Very important is to mention the "added value" that one has when one collects THE SAME data in a long time period (i.e. several years). In this case one can produce "derived results" such as time analyses (e.g. regression lines predicting trends in time). It is therefore strongly advisable, once one has reached an agreement on the definition of a topic, to keep this definition fixed. Otherwise one cannot make comparisons it time. Of course, at any time, one needs to introduce new statistics e.g. on new technologies (CD-ROM, Internet use and so on).

The building of a good data collection takes a long time. It is clear that if one decides in year $\mathbf{x}$ to report on a new topic, one can only start collecting in year $\mathbf{x}+1$, hence reporting on this topic can only start in year $\mathbf{x}+2$. Add to this several more years in order to have a time dimension in these data!

〈heading 2〉II.4 Examples of data

We will give some possible topics that can be collected in a library. I base myself on data that are collected in my library (a small university library, mainly consisting of literature in the exact and medical sciences and in applied economics). The list is only mentioned as an example (to give some hints) and should not be considered as minimal nor as maximal !

〈heading 3)II.4.1 Data on the collection

number of books ordered, catalogued, invoiced, possibly divided over several subjects, including the budgets spent. The same goes for journals, series, CD-ROM products, other multimedia publications and so on. Make clear if series are counted in the books data or not. Compare e.g. medecine with other subjects,

binding issues (journals, restauration, ...).

〈heading 3/II.4.2 Data on user services

on opening hours, giving also statistics on the use of the library in the different periods that the libary is open (e.g. in the evening),

circulation data (incl. renewals, new borrowings, books returned or reserved) and all administration involved with this. Distinguish between the different types of borrowers and different types of lended material. This can e.g. prove that medical staff members or students do not use the library so often and then one can search for an explanation of this and propose possible solutions,

interlibrary data, both on incoming or outgoing requests and both on lending (of books) or copy (of articles) aspects. Calculate the success rates (the quotient of the number of satisfied requests and the total number of requests). Inform about the importance of each used library in this. Try to inform on the speeds involved (of each library), 
photocopy service, printing service (of material from a computer),

use of OPAC : total and average session time. These data can yield conclusions to what extent the OPAC is used as a bibliographic database,

use of external databases online (commercial hosts or Internet) or on CD-ROM. Compare the use of similar databases (e.g. EMBASE versus MEDLINE) in order to decide on future uses. Calculate all costs and try to make distinction between the type of library user. We make here the remark that often can be made : one should be able to prove the special situation of health libraries in many of these topics so that this knowledge can be used wherever it is appropriate,

library facilities and maintenance : number of seats, number of books reshelved, number of stolen or misshelved books and so on.

〈heading 3〉II.4.3 Automation and catalogue aspects

- $\quad$ if this is done in a network, then briefly report on the profile of the partners and situate your library in this,

indicate the total use of the system.

〈heading 3)II.4.4 Staff aspects

describe the employment in persons and in FTEs, inluding the help of job students, draw an organisational chart.

$\langle$ heading 3〉II.4.5 External relations

report on activities outside the library : interlibrary meetings, network activities, talks, conferences, ....,

report on possible scientific research, incl. publications as books or in journals or conference proceedings.

〈heading 3)II.4.6 Budgets

specific budgets should be mentioned at their proper location. The general budget can be mentioned here.

〈heading 3〉II.4.7 Data on companies involved in the library's activities

measurements on the quality (speed, price, ...) of booksellers or journal intermediaries,

quality of the binding company, companies involved with maintenance of PCs, photocopying machines, furniture, etc. 


\section{〈heading 1)III. The use of the data}

So far I have only described the collection of "raw data". This is indeed a very important aspects of reporting quantitatively about the library. These data form the building blocks for later use. But in the same way as building blocks for a house are important but not of any value without proper use, the same can be said about raw data : they need to be streamlined, summarised and described is a clear way, incl. conclusions and interpretations. These activities then form the basic ingredients for the diverse reports that are made.

We make distinction between integral counts (I) and non integral counts (NI) as described in section II.1. Indeed the use is completely different as we will explain further.

〈heading 2)III.1 The use of complete data (of integral counts)

Here we have determined the universe (i.e. the total population on what we want to report on) and we are able to investigate EVERY member of this population. Then our conclusions are $100 \%$ sure, but of course limited to this population. Examples were given in section II.1.

These data are summarised in graphs, such as bar diagrams (for discrete data) and histograms (for continuous data). Examples of discrete data are : the age (in years) of a book or the number of years that a book is in the library, number of authors of medical books, number of library users and so on. Examples of continuous data are : the thickness of medical books (e.g. in $\mathrm{cm}$ ), retrieval times (in minutes or seconds) in an OPAC, CD-ROM or online system, delivery times (in days) of books that are ordered at a book seller. Note that the division between discrete data and continuous data is sometimes vague : delivery times are discrete data (i.e. entire numbers of days) but we are only interested in them in groups, say periods of 5 or 10 days in order to estimate the order of magnitude. Ages in years are clearly discrete while retrieval times in seconds are clearly continuous !

Important, as mentioned before, is the time dimension in the reports. Of course, to establish this we need data of several different time periods (e.g. several years - say at least 6 or 7). In order to visualise the "trend" in time we can calculate the so-called regression line : on the graph consisting of the different points (with the $x$-axis representing time $t$ ) we add a straight line, fitting the "cloud of points" in the best way. By doing so we can see the trend by visual inspection of the line and are able to make (short term) predictions. For an illustration of this, see Fig. 1.

For more on how to make graphs (and also on how NOT to make graphs) we refer the reader to the very interesting and easily readable book [2].

Besides graphs, the so-called "summary statistics" are also needed to interpret the data in a professional way. There are many but the basic ones are : the mean (average) denoted by $\mu$, the standard deviation (dispersion) denoted by $\sigma$ and the so-called percentiles. The square of $\sigma$ is called the variance. The use of the average is clear : it gives an overall overview of the data, e.g. the average price of books. Such a number allows to estimate the total number of books that we can order, given a certain amount of money. The standard deviation and the percentiles yield information about the degree of irregularity of the data. $\sigma$ is mainly used 
in the cases that the data are incomplete. Percentiles are used in complete data, e.g. in the description of delivery times of materials such as ordered books at a bookseller or interlibrary materials at certain libraries. They give answers to questions such as : how long does it take to deliver 25,50 or $75 \%$ of the books that I have ordered at a certain bookseller?

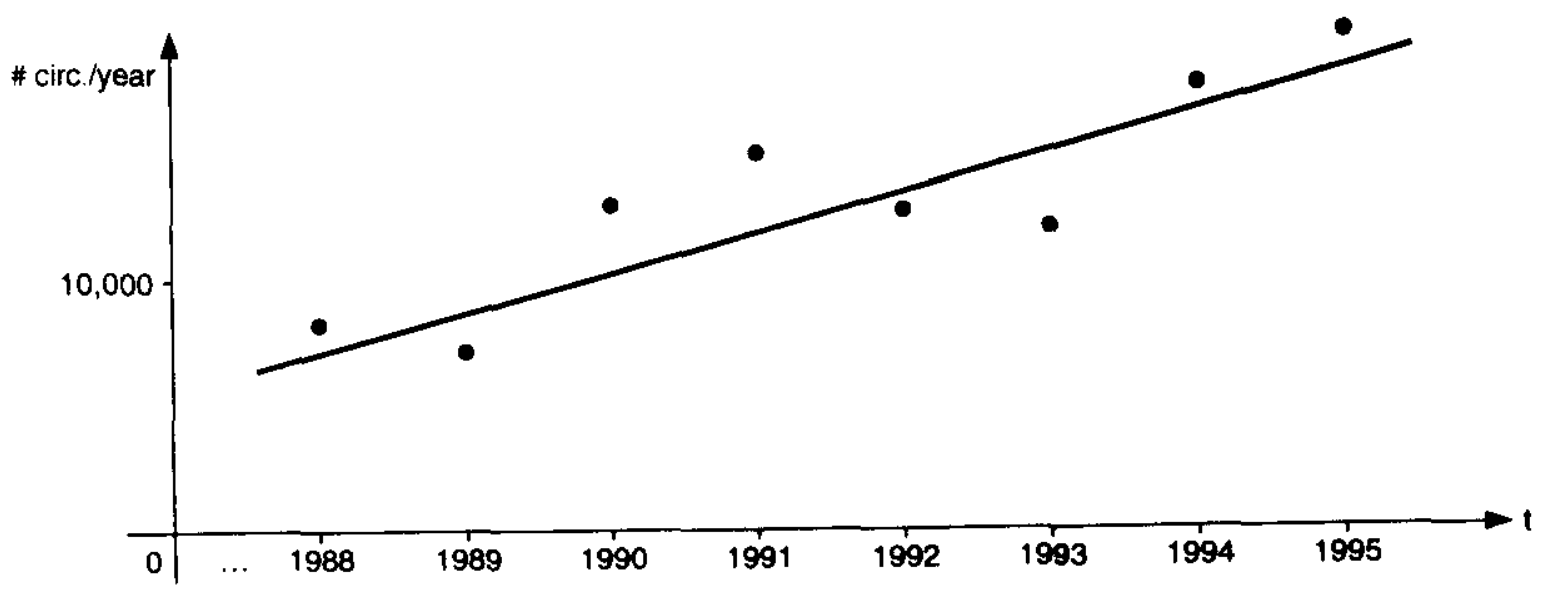

Fig. 1. Scatterplot : Number of circulations/year in function of the year.

〈heading 2)III.2 The use of incomplete data

〈heading 3)III.2.1 The case of one data set

The possible techniques explained in section III. I can, of course, be used in case our data are incomplete but it is evenly clear that at that time we are only reporting on the incomplete data. Let us illustrate this by an example. Suppose that we sampled 100 books, delivered by a bookseller and the we calculate the average delivery time (say 61 days). We can make a complaint to this bookseller hereby requesting a faster delivery. The bookseller can tell us that we sampled only 100 books out of 2.000 deliveries. hence he/she can question the validity of our data. We can solve this problem by giving an estimate of the real average delivery time of this bookseller : we calculate the so-called confidence interval. Suppose our sample had size $\mathbf{N}$ (100 in our example), average $x$ and standard deviation $s$. Then we are $95 \%$ sure that the real "overall" average delivery time of this bookseller is in the interval

$$
\left[\bar{x}-1.96 \frac{s}{\sqrt{N-1}} ; \bar{x}+1.96 \frac{s}{\sqrt{N-1}}\right]
$$


Example : $\mathrm{N}=100, \mathrm{x}=61$ days and $\mathrm{s}=18$ days. Then the real average delivery time is between the values 57.5 and 64.5 days (with $95 \%$ certainty).

Other applications : thickness of medical books, length of reference lists of medical articles, number of authors of medical books. In the area of user studies (very important nowadays) : fraction (fractions are averages - why ?) of certain types of users of the library, fraction of users who agree with a change of the opening hours, fraction of the loan transactions containing the maximum number of books that are allowed to check out at one occasion, fraction of books that are returned too late, fraction of lost/stolen books, fraction of users that are interested in an SDI-service on the acquisitions of the health library. Finally we mention another important application : measuring the overlap between two libraries or between two databases : $\mathrm{O}(\mathrm{B} / \mathrm{A})=$ the overlap of database $\mathrm{B}$ w.r.t. A (i.e. the fraction of the articles in $\mathrm{A}$ that are also in $\mathrm{B})$.

\section{〈heading 3/III.2.2 The case of two data sets}

It is an interesting issue to compare two populations. For example to compare two libraries w.r.t. to their speed of delivery of interlibrary requests. It is clear that, as in subsection III.2.1 we have here two incomplete data sets ! What can we say about the difference ? Analogous as in the previous subsection there are techniques in statistics that allow us to draw $95 \%$ or $99 \%$ (say) conclusions about the possible different behaviour.

Examples of application : difference between the delivery times for books at two booksellers or of books coming from different countries (or, as mentioned above, of interlibrary materials coming from different libraries), difference between the average thickness of medical books and of mathematics books, difference between their average number of authors, fraction of female users of library A versus the same in library B. Final general example : difference of use when we consider two different time periods (measuring changes in quality or in use of certain services).

\section{〈heading 2$\rangle$ III.3 A word on the establishment of incomplete data}

So far we have discussed incomplete data without mentioning how to collect them. Indeed this is a nontrivial problem. By the very definition of "incompleteness", we are faced with the problem : which elements of the population are we going to use in our sample ? The main problem is the possible bias, that is to give to the elements of the population an unequal chance to be picked.

Examples of biasses : to give a questionnaire only to library users that are in the library (in this way you might miss the unsatisfied absent users), picking books in the library shelf by measuring lengths (this way thicker books have a higher chance to be picked), checking the length of services by sampling say every 30 minutes, and so on.

There is a perfect method in order to sample correctly : the method of random sampling. Here we give, to every element of the population, an equal chance to be picked. The method uses a computer generated list of random numbers and they determine the elements of the population that should be picked (for materials that are in the computer this random sampling can be executed in an automatic way). Executing random sampling in a manual way uses lots of time. There exist faster (and often almost as good as random sampling) methods (e.g. the 
Fussler sampling technique). For this, the reader is referred to [3,4] or to the vast statistical literature. These references can also be used for obtaining more information on all the other aspects that are discussed in this paper.

\section{〈heading 2)III.4 Biomedical exercises}

\section{〈heading 3/III.4.1 Research of a student in marine science librarianship}

In the connection of a development project, funded by the Belgian government and executed by LUC (the project manages marine science information for the East-Africal region - see [5] for a recent report on its activities and results), a study visit was executed in 1995 by one of the Kenian scientific assistants in the project (Mr. K. Onyancha). One of his study areas was exactly the topic of this paper. He was interested in the special characteristics of medical books as compared to other scientific books.

Amongst several things he investigated the following properties of medical books :

the number of co-authors of medical books (application : see section IV), the thickness of medical books (application : see below), the age of medical books (application : see section IV).

For the first problem he investigated a sample of 112 medical books from the LUC library and found an average number of co-authors of 1.88. I told him this was interesting in itself but with a little bit of more work he would be able to draw conclusions about the total population of medical books (without investigating them all ; in fact only the small sample is needed). He therefore calculated the standard deviation $\mathbf{s}$ and used the formula in subsection III.2.1 in order to reach the conclusion that there are on average $1.88+/-0.28$ authors per medicine book (with $95 \%$ certainty) in the LUC library. He then did the same for the average thickness of medical books. He reached the answer that the average thickness of medical books in the LUC library is $36.96 \mathrm{~mm}+/-3.25 \mathrm{~mm}$. This type of conclusion is important to plan space and shelving in the library. Finally he did the same for the average age of medical books. Here he found the year $78.5+/-1.5$.

The next question was : are these results different as compared with other disciplines or w.r.t. to the general library characteristics ? If so, then we have proof that special attention must be given to medical collections and, of course, the used methods yield a professional way to inform policy makers about it. As to the first characteristic we studied medical books versus mathematics books. For the latter we found on the average 1.64 co-authors per mathematics book and a test on the difference of two population averages revealed that the difference was indeed significant. The same conclusion could be drawn for the second characteristic (again the larger number for medical books) but not for the third one.

\section{〈heading 3)III.4.2 Results from commons statistics in a library consortium}

I could report here on several other statistical aspects on the use of the biomedical literature in the LUC library (for this, see the annual report of the LUC library, [6]) but I prefer to mention a study that was carried out last year in the VOWB (Flemish Council on Scientific Library Aspects), comprising e.g. all Flemish university libraries on the strength/weakness of the diverse library collections. Amongst many things I found that the medical journal 
collection of my library is unacceptably small in the following sense. One had calculated for each library - the percentage of the titles (appearing in the consortium of university libraries) that belong to this library. To my surprise the score of LUC was the lowest in medecine, even lower than in the human or applied sciences which are directions in which LUC does not offer teaching (and this in contrast to the medical sciences) ! It is clear that such reports are more powerful than any series of numbers that I can derive from my library alone. In fact these results were considered as very important by the academic leaders of LUC ! For more on this we refer to [7].

\section{〈heading 1)IV Other bibliometric techniques, referring to the case of biomedical literature}

The medical sciences are one of the best disciplines to evaluate the research output of scientists. The method is based on citation analysis : it measures the amount of citations that a medical article (that appeared in a so-called source journal) receives after it is published. The basic data can be found in the SCI (Science Citation Index), a product of ISI (Institute of Scientific Information) and in its side product, the JCR (Journal Citation Reports).

At the occasion of the writing of this paper I checked the LISA and ISA database for literature on "medical bibliometrics". It was immediately clear that the vast majority of the publications are in this "citation analysis" area (mainly appearing in the journal Scientometrics but also in more "medically oriented" library journals such as the Bulletin of the Medical Library Association). Medical scientists should know that the "visibility in the world" of their research is measured in this way (by their proper institution but also by several subsidising bodies such as National Science Foundations or governments) and hence it is best that they should be informed about these techniques.

Citation analysis also yields a powerful tool for the health information manager to estimate the research degree of journals : the higher its so-called impact factor (the relative number of times an article in this journal is cited by source journals in a 2 year period), the higher its use and its visibility and hence (to a large content) its research degree and research quality.

Citation analysis can also be used to estimate obsolescence (also called ageing) of literature in a research area. This is because references at the end of a paper also contain a date indication. Ageing data on scientific journals can help the librarian in his/her acquisition policy (insofar the librarian has an influence in this). It is one of the elements (the price of a journal obviously is another element of course) in the decision on whether a journal should be bought or not (also in comparison with interlibrary requests for this journal - again incl. the age of the requested materials). This method was followed in the above mentioned development project on marine science literature in East-Africa (cf. [5]).

Ageing is the diminishing use in time of literature. Growth is the increased production in time of literature. At first sight ageing and growth do not have any relationship nor influence on each other. This is not true. First of all, the techniques to study ageing and growth are exactly the same. In both cases one calculates a so-called "rate" function, being the amount in year $\mathrm{t}+1$ divided by the amount in year t. Here "amount" means : "number of citations" 
for the ageing case or "number of publications" for the growth case. Secondly there is an influence of growth on obsolescence. For this we refer the reader to the articles [8-11]. $[12,13]$ are two sample references on growth of biomedical literature.

We finally remark that many techniques used by scientometricians are based on multivariate statistics. This method consists of mapping a high dimensional space onto a "preferred" plane so that natural groupings and interrelationships between the points become clear. The points in this high dimensional plane are obtained by the formation of a matrix of data linking a set of objects to a set of veriables (the number of objects determines the number of points ; the number of variables determines the number of dimensions). Two examples :

- both objects and variables are journals and the link between them is the number of times one journal cites another. A variant consists of linking two journal articles if they are co-cited a certain number of times. In these cases "atlasses" of the discipline can be constructed and subdisciplines generated automatically. [14] is an interesting recent reference on this.

both objects and variables are authors and the link between them is the number of times they are co-authors. In this case scientific "schools" are generated (or more simply, research groups). This in turn is useful in the evaluation of the scientific output of research groups. As a recent sample reference we refer the reader to [15] and also to [16].

We refer to [17] for an example on the clustering of pharmacological journals.

For more on these and other bibliometric techniques, the reader is referred to $[3,4]$.

\section{References}

[1] ARL Statistics (1995), ARL Statistics 1993-94. A compilation of statistics from the one hundred and nineteen members of the Association of Research Libraries. Association of Research Libraries, Washington, D.C.

[2] Cleveland W.S. (1985), The Elements of graphing Data. Wadsworth, Monterey, California, USA.

[3] Egghe L. and Rousseau R. (1990), Introduction to Informetrics. Quantitative Methods in Library, Documentation and Information Science, Elsevier, Amsterdam.

[4] Egghe L. and Rousseau R. (1996), Management of modern Libraries and multimedia Centres using elementary Statistics. Book, preprint.

[5] Egghe L. and Pissierssens (1996), Managing marine science information in EastAfrica : the RECOSCIX-WIO project. Preprint. 
[6] Universiteitsbibliotheek LUC (1996), Jaarverslag 1995. Unpublished report.

[7] Muyldermans J., Braeckman J. and Bosmans W. (1996), Eindrapport Sterkte/ZwakteAnalyse van lopende Tijdschriftencollecties in tien Vlaamse Wetenschappelijke Bibliotheken. VOWB, Brussel, België.

[8] Egghe L. (1993), On the influence of growth on obsolescence. Scientometrics, 27(2), 195-214.

[9] Egghe L. (1994), A theory of continuous rates and applications to the theory of growth and obsolescence rates. Information Processing and Management, 30(2), 279292.

[10] Egghe L., Rao I.K.R. and Rousseau R. (1995), On the influence of production on utilization functions : obsolescence or increased use ? Scientometrics, 34(2), 285-315.

[11] Rao I.K.R. and Meera B.M. (1992), Growth and obsolescence of literature : an empirical study. Proceedings of the Third International Conference on Informetrics, I.K.R. Rao (ed.), Bangalore, India, August 1991, 377-394.

[12] Humphreys B.L. and McCutcheon D.E. (1994), Growth patterns in the National Library of Medecine's serials collection and in Index Medicus journals, 1966-1985. Bulletin of the Medical Library Association, 82(1), 18-24.

[13] Pratt G.F. (1992), A decade of AIDS literature. Bulletin of the Medical Library Association, 80(4), 380-381.

[14] Duplenko Y.K. and Burchinsky S.G. (1995), Computer-aided clustering of citation networks as a tool of mapping of research trends in biomedecine. Scientometrics, $32(3), 247-258$.

[15] Herbertz H. (1995), Does it pay to cooperate ? A bibliometric case study in molecular biology. Scientometrics, 33(1), 117-122.

[16] Logan E.L. and Shaw W.M.Jr. (1991), A bibliometric analysis of collaboration in a medical specialty. Scientometrics, 20(3), 417-426.

[17] Rousseau R. (1989), Evolution d'importantes revues pharmacologiques. Revue Française de Bibliométrie, 5, 102-117. 